\title{
CYTOMORPHOLOGICAL DETECTION OF MALIGNANT EFFUSIONS IN NON-HODGKIN LYMPHOMA: AN INSTITUTIONAL EXPERIENCE IN A DEVELOPING COUNTRY
}

\author{
BHAVNA NAYAL*, GEETHA V \\ Department of Pathology, Kasturba Medical College, Manipal, Manipal Academy of Higher Education, Manipal, Karnataka, India. \\ Email: bhavna.nayal@manipal.edu
}

Received: 24 August 2018, Revised and Accepted: 10 December 2018

\begin{abstract}
Objective: Lymphomatous effusions of the body cavity may be the presenting feature or develop later as a complication of systemic disease. The detection rate of lymphoma in cytologic specimen is low, especially in the absence of clinical details and ancillary studies. The present study was carried out to identify light microscopic features that are useful in identifying lymphomas on effusion cytology.
\end{abstract}

Methods: A 5-year retrospective study of all patients with fluid cytology or tissue biopsy reported as suspicious or positive for non-Hodgkin lymphoma (NHL) in a tertiary care was done. The cytology, histopathology, and immunohistochemistry slides were reviewed.

Results: A total of 27 cases were included in the study. Correlation with the histopathological sections of all the positive cases revealed that the cytomorphology of the abnormal lymphoid cells was monomorphous and similar to those seen in the tissue biopsy. Mercury drop karyorrhexis when present was characteristic of lymphomatous effusions. The detection rates of large cell lymphomas are higher than low-grade counterparts. Non-lymphomatous effusions showed heterogeneous lymphoid cell population and lacked karyorrhexis.

Conclusion: Lymphomas can give rise to effusions. In the absence of resources in developing countries, it is important to distinguish lymphomatous effusion from a reactive process based on morphology. Monomorphous population of the lymphoid cells and presence of mercury drop karyorrhexis are useful morphological clues in identifying a lymphomatous effusion. Further, tuberculosis is a common non-neoplastic process that can be mistaken for a low-grade NHL.

Keywords: Effusion, Non-Hodgkin's lymphoma, Cytology, Karyorrhexis.

(c) 2019 The Authors. Published by Innovare Academic Sciences Pvt Ltd. This is an open access article under the CC BY license (http://creativecommons. org/licenses/by/4. 0/) DOI: http://dx.doi.org/10.22159/ajpcr.2019.v12i3.29264

\section{INTRODUCTION}

Effusion is a common complication of lymphomas and may develop at any time during the course of the disease. If present at the time of diagnosis, it adversely affects the overall survival and is associated with a higher rate of disease relapse following treatment $[1,2]$.

Cytology is a method commonly employed in the evaluation of effusions. However, sometimes, it may be extremely difficult to differentiate a reactive process from lymphomatous effusions. Immunocytochemistry, flow cytometry, and cytogenetics help in such situations. However, in the absence of clinical details and ancillary studies, the detection rate of lymphoma in cytologic specimen is low, i.e., approximately $10 \%$ [1-3].

The present study was carried out to identify the light microscopic features that are useful in identifying lymphomas on effusion cytology.

\section{METHODS}

A retrospective study was conducted on all patients with fluid cytology or histopathological diagnosis of non-Hodgkin lymphoma (NHL) in our institute between March 2012 and March 2017. Cases where cytology slides were not available or inadequate for opinion were excluded from the study. All available Papanicolaou-stained cytology slides were retrieved from the archives of the department of pathology and reviewed. Based on the histopathological findings, false negative, true negative, and true positive cases were identified. Cytological features studied were heterogeneity in the size of cells, cohesiveness, cell size, presence of karyopyknosis, mercury drop karyorrhexis, mesothelial cells, tingible body macrophages, and lymphoplasmacytoid (LP) cells. These parameters were analyzed and compared in patients with positive and negative cytology.

\section{RESULTS}

A total of 27 cases of effusions were included in the study; 16 were pleural fluid, 10 peritoneal fluid, and one testicular fluid. 13 of these 27 cases were reported as reactive process on cytology. On review, five (false negative) of these 13 were reclassified as malignant with subsequent tissue biopsies showing high-grade lymphoma in all of them. The remaining eight (true negative) cases did not show neoplastic cells in the effusion.

Five cases were reported as suspicious for NHL on cytology. None of these had a tissue biopsy at the time of cytological examination. Three of these were recategorized as NHLs. Biopsy performed subsequently showed two low-grade and one high-grade NHL. The remaining two cases diagnosed suspicious on cytology had Mycobacterium tuberculosis detected by polymerase chain reaction.

Nine cases of lymphomatous effusions were true positive. Five of them were diagnosed on cytology while four were known case of NHLs, diagnosed initially on histopathology. All these cases revealed highgrade morphology histologically.

The cell size in the false negative cases was medium to large in two and small to medium in three cases. Monomorphous lymphoid cell population was strikingly common in the positive cases (Fig. 1).

The presence of MKD gave a false impression of polymorphous population of cells in two cases and one each in the positive and false negative group (Fig. 2). Nuclear irregularities and nucleoli were seen in three of these five cases. These findings were possibly missed or misinterpreted resulting in the false negative report. 
Of the five cases reported as suspicious, four had monomorphous population of small-sized lymphoid cells while one had polymorphous population of small-to medium-sized cells. The latter smear on review revealed KP and MKD along with binucleate forms and was subsequently reported positive. Two cases were also reported positive

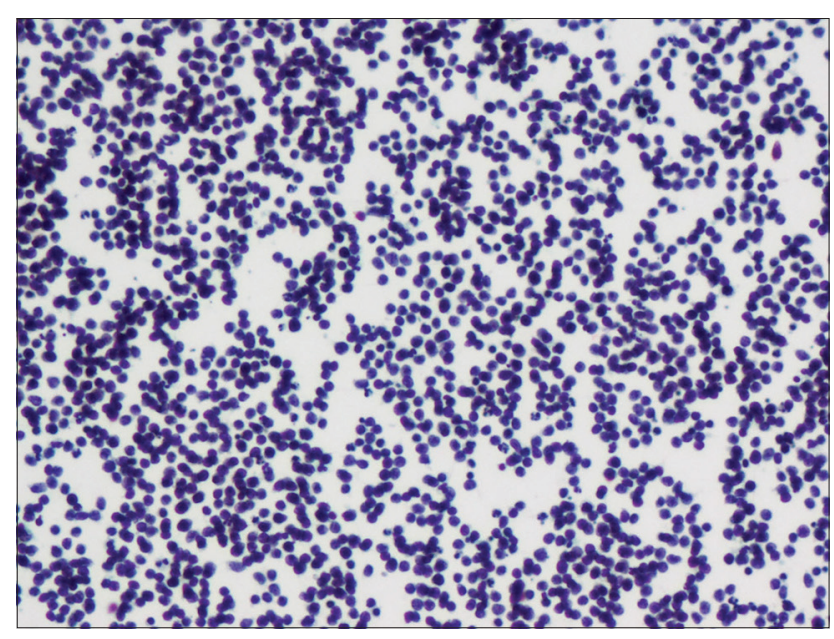

Fig. 1: Monomorphous population of lymphoid cells in lymphomatous effusion (PAP, ×20)

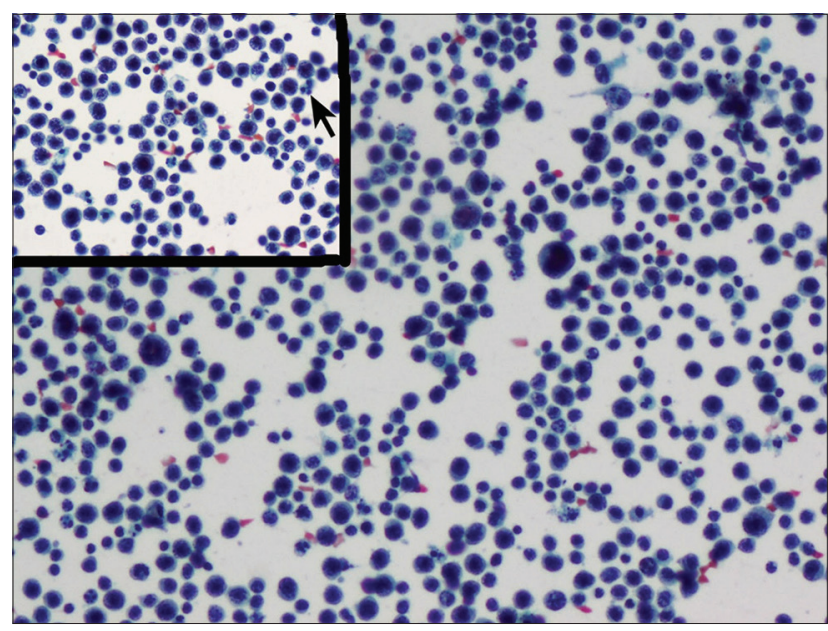

Fig. 2: Polymorphous population of lymphoid cells in lymphomatous effusion $(P A P, \times 20)$ Inset: Arrowed structure: Mercury drop karyorrhexis resembling neutrophil $(\times 40)$ as they showed nuclear irregularities. However, there was the absence of KP and MKD. The remaining two cases were negative.

All these cases were finally classified as positive (17) including nine true positives, five false negatives, and three that were initially diagnosed suspicious. The negative (10) group included the eight true negative cases and two which were suspicious for NHL.

The cytomorphological features of these cases $(n=27)$ are shown in Table 1.

Correlation with the histopathological sections of all the positive cases $(17 / 27)$ revealed that the cytomorphology of the abnormal lymphoid cells was similar to those seen in the tissue biopsy.

The cytology of positive cases showed cells with round-to-oval nuclei with irregular nuclear membranes and one to multiple nucleoli. Five cases showed binucleation. Karyopyknosis (35\%) and MKD (59\%) when present were characteristic of lymphomatous effusions and were seen more often in high-grade lymphomas. These two features were absent in negative cases. The detection rates of large cell lymphomas were higher than their low-grade counterparts.

Non-lymphomatous effusions showed polymorphous lymphoid cell population, lacked karyorrhexis, and demonstrated mesothelial cells (Fig. 3).

\section{DISCUSSION}

Effusions in lymphoma may be malignant, as a part of the disease process, or reactive, secondary to infections due to reduced immunity or therapy. The former can be explained by direct infiltration, lymphatic obstruction, hematogenous dissemination, or widespread disease [4]. Cytological examination of the effusion fluid in lymphoma provides a rapid and accurate diagnosis, with diagnostic accuracy of tissue biopsy and has therapeutic and prognostic implications $[1,2,5]$. In diffuse large B-cell lymphoma, the presence of malignant cells in effusions has been found be a stronger poor prognostic and predictor factor than high stage [6].

While evaluating fluid cytology, the presence of dyscohesive, single isolated cells favors a neoplastic process. The presence of monomorphic population of large lymphoid cells was a characteristic finding useful in the cytological diagnosis of lymphomatous effusions. Other cytologic clues useful in NHL associated effusions include karyopyknosis and MDKs. The presence of tingible body macrophages though uncommon also served as another useful feature. Hence, the higher grade NHLs were detected more often. The absence of monomorphism may contribute to the low detection of low-grade NHLs [2,5,7]. In the present study, all the

Table 1: Cytomorphological features of effusions $(n=27)$

\begin{tabular}{llll}
\hline Morphologic characteristics & \multicolumn{2}{l}{ Positive cytology (n=17), $\mathbf{~ ( \% ) ~}$} & Negative cytology (n=10), $\mathbf{n}(\mathbf{\%})$ \\
\cline { 2 - 3 } & \multicolumn{1}{l}{ High grade (n=15) } & Low grade (n=2) & \\
\hline Cell population & $7(41)$ & $1(6)$ & 0 \\
$\quad$ Monomorphous & $8(47)$ & $1(6)$ & $10(100)$ \\
$\quad$ Polymorphous & $15(88)$ & $2(12)$ & $10(100)$ \\
Dyscohesive cells & & $2(12)$ & $10(100)$ \\
Cell size & 0 & 0 & 0 \\
Small & $6(35)$ & 0 & 0 \\
Small to medium & $1(6)$ & 0 & 0 \\
Medium & $5(29)$ & 0 & 0 \\
Medium to large & $3(18)$ & 0 & $2(20)$ \\
Large & $6(35)$ & 0 & 0 \\
Karyopyknosis & $10(59)$ & 0 & $7(70)$ \\
MDK & $2(12)$ & 0 & 0 \\
Mesothelial cells & $3(18)$ & 0 & $1(10)$ \\
Tingible body macrophages & $3(18)$ & & \\
Lymphoplasmacytoid cells & &
\end{tabular}




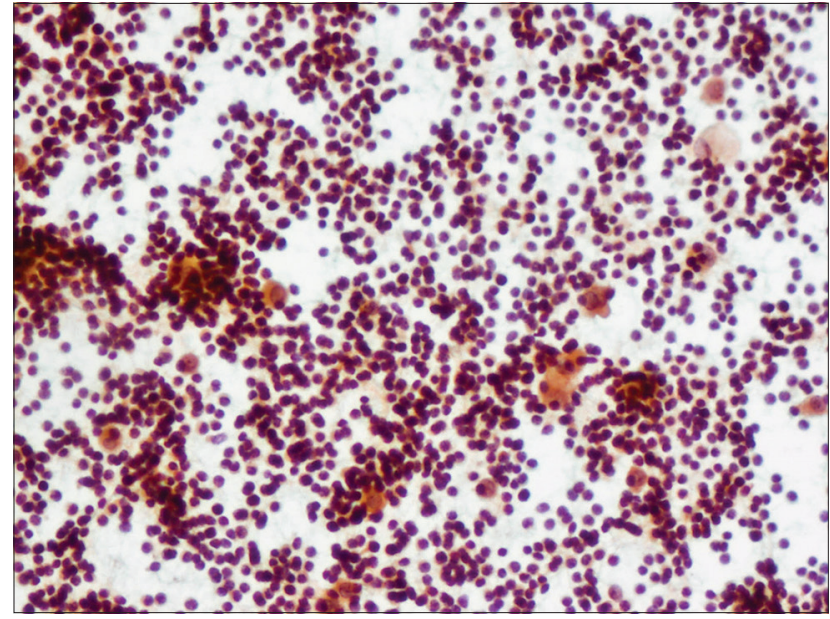

Fig. 3: Polymorphous population of lymphoid cells in reactive effusion demonstrating mesothelial cells $(\mathrm{PAP}, \times 20)$

negative cases had polymorphous population of lymphoid cells with the absence of karyopyknosis and MDKs.

Cell necrosis in the form of fragmented nuclei with small round cyanophilic cytoplasmic particles called mercury drop karyorrhexis (MKD) may be mistaken for neutrophils, giving a false heterogeneous appearance to the cell population in a lymphomatous effusion, especially in low power examination [8]. MKD was present in 59\% of positive cases and was absent in negative cases. Hence, this is a useful feature to differentiate neoplastic from inflammatory effusions.

There is usually a paucity of stimulated or reactive lymphoid cells including LP cells in reactive effusions. The presence of $>10 \%$ stimulated lymphoid cells in an effusion should raise a suspicion for malignancy. Three of 17 positive cases (18\%) showed LP cells in the present study [9].

Cytological characteristic of the lymphoid cells is an important indicator of lymphomatous effusions. Effusions in high-grade lymphomas were more readily detected due to the presence of medium to large cells with irregular nuclei, coarse chromatin, prominent nucleoli, karyopyknosis, and frequent mitosis. However, it was difficult to distinguish neoplastic cells in low-grade lymphoma from benign lymphocytes in reactive effusions which are also small sized with regular nuclear contour and inconspicuous nucleoli. The presence of polymorphous population of small-sized cells in the latter was the clue for excluding lymphomatous effusions in these cases.

In our country, a common non-neoplastic cause of effusion, which shows predominance of lymphoid cells, is tuberculosis. Biochemical clues such as elevated fluid protein and adenosine deaminase levels can be seen in both tuberculous and lymphomatous effusions. Effusions due to tuberculosis also show sparse mesothelial cells $(<5 \%)$ which is a common feature of lymphomatous effusions [10-12]. Hence, these biochemical and cytological overlaps contributes to the diagnostic difficulty in distinguishing between the two.

In the present study, all the false negative cases were of effusions from high-grade lymphoma. On review, these effusions appeared to be polymorphous on low power examination due to numerous MKD and fewer viable tumor cells in between them. The presence of karyopyknosis, nuclear irregularities, and paucity of background mesothelial cells helped to identify the neoplastic effusions. Further, the monomorphous small-sized cells in the effusions reported as suspicious were identified as positive due to the nuclear characteristics [13].

The major limiting factor in the present study is the small sample size of the study group due to which adequate statistical analysis cannot be done.

\section{CONCLUSION}

Early and rapid identification of the involvement of body cavities is essential for prompt and appropriate treatment. It is challenging but very important to distinguish lymphomatous effusion from a reactive process based on cytomorphology in the absence of modern ancillary tests in resource poor. Cytomorphological features favoring lymphomatous over reactive effusions include monomorphous singly placed lymphoid cells with nuclear indentations/protrusions, prominent nucleoli, and mitosis. A background containing nuclear fragmentation, karyorrhexis, and absence or sparse mesothelial cells can also serve as cytologic clues to initiate ancillary testing. However, similar studies with larger cohort are warranted to ascertain statistical significance.

\section{ACKNOWLEDGMENT}

Nil.

\section{AUTHORS' CONTRIBUTIONS}

Dr. Bhavna Nayal - Collection of data and manuscript writing. Dr. Geetha V - Data analysis and manuscript editing.

\section{CONFLICTS OF INTEREST}

Nil.

\section{REFERENCES}

1. Das DK. Serous effusions in malignant lymphomas: A review. Diagn Cytopathol 2006;34:335-47.

2. Bangerter M, Hildebrand A, Griesshammer M. Combined cytomorphologic and immunophenotypic analysis in the diagnostic workup of lymphomatous effusions. Acta Cytol 2001;45:307-12.

3. Storey DD, Dines DE, Coles DT. Pleural effusion. A diagnostic dilemma. J Am Med Assoc 1976;236:2183-6.

4. Alexandrakis MG, Passam FH, Kyriakou DS, Bouros D. Pleural effusions in hematologic malignancies. Chest 2004;125:1546-55.

5. Bode-Lesniewska B. Flow cytometry and effusions in lymphoproliferative processes and other hematologic neoplasias. Acta Cytol 2016;60:354-64.

6. Chen YP, Huang HY, Lin KP, Medeiros LJ, Chen TY, Chang KC, et al. Malignant effusions correlate with poorer prognosis in patients with diffuse large B-cell lymphoma. Am J Clin Pathol 2015;143:707-15.

7. Liam CK, Lim KH, Wong CM. Differences in pleural fluid characteristics, white cell count and biochemistry of tuberculous and malignant pleural effusions. Med J Malaysia 2000;55:21-8.

8. Melamed MR. The cytological presentation of malignant lymphomas and related diseases in effusions. Cancer 1963:16:413-31.

9. Spriggs AI, Vanhegan RI. Cytological diagnosis of lymphoma in serous effusions. J Clin Pathol 1981;34:1311-25.

10. Cakir E, Demirag F, Aydin M, Erdogan Y. A review of uncommon cytopathologic diagnoses of pleural effusions from a chest diseases center in Turkey. Cytojournal 2011:8:13.

11. Antonangelo L, Vargas FS, Genofre EH, Oliveira CM, Teixeira LR, Sales RK, et al. Differentiating between tuberculosis-related and lymphoma-related lymphocytic pleural effusions by measuring clinical and laboratory variables: Is it possible? J Bras Pneumol 2012;38:181-7.

12. Light RW. Update on tuberculous pleural effusion. Respirology 2010;15:451-8.

13. He SR, Peng WX, Sun MJ, Yang L, He L, Su XL, et al. Cytodiagnosis and cytomorphologic analysis of hematolymphoid malignancy in serous effusion: A study of 23 cases. Zhonghua Bing Li Xue Za Zhi 2012;41:691-5. 